\title{
Why entanglement of formation is not generally monogamous
}

\author{
F. F. Fanchini, ${ }^{1,}{ }^{*}$ M. C. de Oliveira, ${ }^{2,3, \dagger}$ L. K. Castelano, ${ }^{4}$ and M. F. Cornelio ${ }^{5}$ \\ ${ }^{1}$ Faculdade de Ciências, UNESP-Universidade Estadual Paulista, Código de Endereçamento Postal 17033-360, Bauru, São Paulo, Brazil \\ ${ }^{2}$ Instituto de Física Gleb Wataghin, Universidade Estadual de Campinas, Código de Endereçamento Postal 13083-859, \\ Campinas, São Paulo, Brazil \\ ${ }^{3}$ Institute for Quantum Information Science, University of Calgary, Alberta, Canada T2N 1N4 \\ ${ }^{4}$ Departamento de Física, Universidade Federal de São Carlos, Código de Endereçamento Postal 13565-905, São Carlos, São Paulo, Brazil \\ ${ }^{5}$ Instituto de Física, Universidade Federal de Mato Grosso, Código de Endereçamento Postal 78060-900, Cuiabá, Mato Grosso, Brazil
}

(Received 7 November 2012; published 13 March 2013)

\begin{abstract}
Unlike correlation of classical systems, entanglement of quantum systems cannot be distributed at will: if one system $A$ is maximally entangled with another system $B$, it cannot be entangled at all with a third system $C$. This concept, known as the monogamy of entanglement, is manifest when the entanglement of $A$ with a pair $B C$ can be divided as contributions of the entanglement between $A$ and $B$ and $A$ and $C$, plus a term $\tau_{A B C}$ involving genuine tripartite entanglement and so expected to be always positive. A very important measure in quantum information theory, the entanglement of formation (EOF), fails to satisfy this last requirement. Here we present the reasons for that and show a set of conditions that an arbitrary pure tripartite state must satisfy for the EOF to become a monogamous measure, i.e., for $\tau_{A B C} \geqslant 0$. The relation derived is connected to the discrepancy between quantum and classical correlations, $\tau_{A B C}$ being negative whenever the quantum correlation prevails over the classical one. This result is employed to elucidate features of the distribution of entanglement during a dynamical evolution. It also helps to relate all monogamous instances of the EOF to the squashed sntanglement, an entanglement measure that is always monogamous.
\end{abstract}

DOI: 10.1103/PhysRevA.87.032317

PACS number(s): 03.67.Mn, 03.65.Ud

\section{INTRODUCTION}

The concept of the monogamy of an entanglement measure $\mathcal{E}$ asserts that, in a tripartite $A, B$, and $C$ system, the entanglement of $A$ with $B C$ can be divided as $\mathcal{E}_{A \mid B C}=\mathcal{E}_{A \mid B}+$ $\mathcal{E}_{A \mid C}+\tau_{A B C}$, where $\mathcal{E}_{A \mid i}, i=B, C$, is a bipartite entanglement and $\tau_{A B C}$ is a genuine tripartite entanglement. In that sense, unlike correlation in classical systems, for entanglement there is a trade-off between the amount of bipartite entanglement $A$ can share with $B$ and $C$. In 2000, Coffman, Kundu, and Wootters (CKW) [1] derived a monogamous relation for the squared concurrence and defined the genuine tripartite entanglement as the tangle (hereafter called the concurrence tangle) [1], $\tau_{A B C}=C_{A(B C)}^{2}-C_{A B}^{2}-C_{A C}^{2}$, where $C_{i j}^{2}$ is the square of the concurrence between the pair $i$ and $j$. The concurrence tangle is always positive for a three-qubit system [1] and for multiqubit systems [2]. However, it is known that a similar analysis made with the entanglement of formation (EOF) would give a tangle that can be positive or negative (hereafter we call this tangle the EOF tangle). Although there are some instances in which the EOF could be distributed in a monogamous fashion, it is known that it is not, in general, a necessarily monogamous entanglement measure (see a more complete discussion in Refs. [3-5]). This is puzzling, since the EOF satisfies many of the axioms required for a good entanglement measure and, further, has a clear operational meaning [6]. So why is the EOF tangle negative or positive?

In fact it is now known that entanglement is not the only form of quantum correlation, since there are instances where a state that is separable (not entangled) still possesses a sort

\footnotetext{
*fanchini@fc.unesp.br

†'marcos@ifi.unicamp.br
}

of correlation which, in principle, could be used to perform certain tasks more efficiently than with classical correlation only. It is not surprising though that both forms of quantum correlation can be related to each other through extended system [7] distribution formulas. For example, it is possible to describe a conservation relation [8] for distribution of the EOF and quantum discord (QD), a measure of quantum correlation; for an arbitrary tripartite pure system, the sum of the QD of a chosen partition, given measurements on the complementary partitions, must be equal to the sum of the pairwise EOF between the chosen partition and the complementary ones. Surprisingly, the sum of the pairwise EOFs appears in a fashion quite similar to the desired expression for the so-called monogamy of entanglement, and the relation obtained can be connected to the way that classical correlations $[9,10]$ are distributed [11-13].

Until a few years ago, the conjecture that the classical correlation would always be greater than the quantum correlation for any quantum state was broadly accepted $[9,10]$. In 2009, Maziero et al. [14] presented the first counterexample to this conjecture, while studying the dissipative dynamics for two qubits. Despite their findings, the balance between classical and quantum correlation has not been connected to any quantum measure or protocol. In this paper, we show necessary and sufficient conditions for the monogamy of the EOF to be established with the help of a general quantum correlation measure, the QD, and identify the EOF tangle for an arbitrary tripartite state as the difference between classical and quantum correlations. We show that the balance between classical and quantum correlation is crucial to understand this important open problem. For that we develop an operational interpretation of the EOF tangle as a measure of the imbalance of the quantum and the classical correlations, here called the correlation discrepancy. In fact, very recently, Giorgi showed 
that the EOF and QD obey the same monogamous relation [12]. So the reasons for the monogamy of the EOF can be extended for the monogamy of the QD as well. Also, Prabhu et al. [13] studied the conditions for monogamy of the QD for the Greenberger-Horne-Zeilinger (GHZ) and the $W$ states and showed that the GHZ family are monogamous while the generalized $W$ states are not. In the present work instead we focus on the distribution of the EOF and give strong bounds in terms of quantum conditional entropies, in an arbitrary tripartite system.

The monogamous relation for the EOF helps in understanding when this measure of entanglement is connected with the squashed entanglement [15]. We give in a simple picture an interesting connection between monogamous instances of both the EOF and QD and the squashed entanglement for arbitrarily tripartite mixed states. The paper is divided as follows. In Sec. II we derive the main relations for understanding the monogamous instances of the EOF. In Sec. III we extend the EOF tangle to a permutation-invariant form, and in Sec. IV we derive some important results for classes of states in $(2 \times 2 \times N)$-dimensional Hilbert space in general and employ it to understand the interplay between bipartite and multipartite entanglement in the phenomenon of sudden death of entanglement. In Sec. V we sketch an extension for arbitrarily mixed states and show how monogamous instances of the EOF are connected to the squashed entanglement. Finally in Sec. VI a discussion closes the paper.

\section{CONDITIONS FOR MONOGAMY OF THE EOF}

Although there is a common understanding that correlation of classical systems can be distributed at will [1], a direct quantification of that is necessary. When thinking of correlation between two stochastic variables $X$ and $Y$, the mutual entropy of Shannon $H(X: Y) \equiv H(X)+H(Y)-H(X, Y)$ is the optimal measure. It turns out that the mutual information is not always subadditive, i.e., $H(X: Y, Z) \not H(X: Y)+$ $H(X: Z)$. But now let us suppose that we can increase $H(X: Y)$ to be maximal [assuming $H(X) \leqslant H(Y)$ ] so that $H(X: Y)=H(X)$. It means that $H(X: Z)=H(X: Y)-$ $H(X \mid Z)$, and so increases linearly with $H(X: Y)$, being constrained only by $H(X: Z) \leqslant H(X: Y)$. When extended to quantum systems, in terms of the von Neumann entropy, the mutual information is written $S(A: B) \equiv I_{A B}=S_{A}+$ $S_{B}-S_{A B}$ and the best we can do to infer the distributon of correlation is to write $S(A: C)=S(A: B)+S(A \mid B)-$ $S(A \mid C)$, with surprising consequences, since the conditional entropy can be negative whenever there is some entanglement in the parties involved. For example, if the joint system $A B C$ is pure, then $S(A: C)=S(A: B)+2 S(A \mid B)=S(A:$ $B)-2 S(A \mid C)$. Also $S(A: B, C) \not S(A: B)+S(A: C)$, but is nonetheless additive if $A B C$ is pure. The mutual information quantifies both the classical and quantum correlation.

From a different point of view, the classical correlation $[9,16]$ in a quantum state can be captured by

$$
J_{A B}^{\overleftarrow{A}}=\max _{\left\{\Pi_{k}\right\}}\left[S_{A}-\sum_{k} p_{k} S_{A \mid k}\right]
$$

where $S_{A \mid k}$ is the conditional entropy after a measurement in $B$. Explicitly $S_{A \mid k} \equiv S\left(\rho_{A \mid k}\right)$, where $\rho_{A \mid k}=$ $\operatorname{Tr}_{B}\left(\Pi_{k} \rho_{A B} \Pi_{k}\right) / \operatorname{Tr}_{A B}\left(\Pi_{k} \rho_{A B} \Pi_{k}\right)$ is the reduced state of $A$ after the outcome $k$ in $B$ and $\left\{\Pi_{k}\right\}$ is a complete set of positive-operator-valued measurements resulting in the outcome $k$ with probability $p_{k}$. Since a measurement might give different results depending on the basis choice, a maximization over $\left\{\Pi_{k}\right\}$ is required. Thus $J_{A B}^{\leftarrow}$ is the maximal locally accessible mutual information, or the maximum amount of $A B$ mutual information that one can get by performing only local measurements on $B$ [17]. On the other hand, quantum discord, as a measure of quantum correlation, gives the amount of locally inaccessible mutual information, and so it is simply defined as

$$
\delta_{A B}^{\leftarrow}=I_{A B}-J_{A B}^{\leftarrow}
$$

The equation above gives the amount of information that is not locally accessible by measurements on $B$ [17]. It is important to emphasize that the quantum discord is asymmetric, since the amount of locally inaccessible information for $B$ can be different from the locally inaccessible information for $A$. This means that generally $\delta_{A B}^{\leftarrow} \neq \delta_{B A}^{\leftarrow}$. Since $I_{A B}$ can be regarded as the mutual information previous to a measurement, while $J_{A B}^{\leftarrow}$ is the mutual information accessed by local measurements in $B$, we can identify $\delta_{A B}^{\leftarrow}$ as a measure of how much a state $\rho_{A B}$ is affected by local measurements on $B$.

It is also convenient to define another quantity measuring the quantum and the classical correlation imbalance, or the correlation discrepancy, or simply the discrepancy, as

$$
\Delta_{A B}^{\leftarrow} \equiv J_{A B}^{\leftarrow}-\delta_{A B}^{\leftarrow}
$$

This measure has the advantage of bearing a clear meaning: It is the balance between the locally accessible mutual information and the locally inaccessible mutual information [11]. Similarly to quantum discord and classical correlation, the discrepancy is an asymmetric quantity and, unlike the former, it can be negative or positive. Actually, if $\Delta_{A B}^{\overleftarrow{ }}>0$ the amount of classical correlation is larger than the quantum one, and vice versa. The discrepancy is bounded by the mutual information as $-I_{A B} \leqslant \triangle_{A B} \leqslant I_{A B}$. Moreover, the discrepancy is strictly zero if the bipartite quantum state is pure since, in that case, the quantum and classical correlations are both equal to $S_{A}$ (see the illustration in Fig. 1). It is interesting to interpret the discrepancy in terms of classical and quantum Maxwell demons $[17,18]$. Quantum demons have the ability to extract

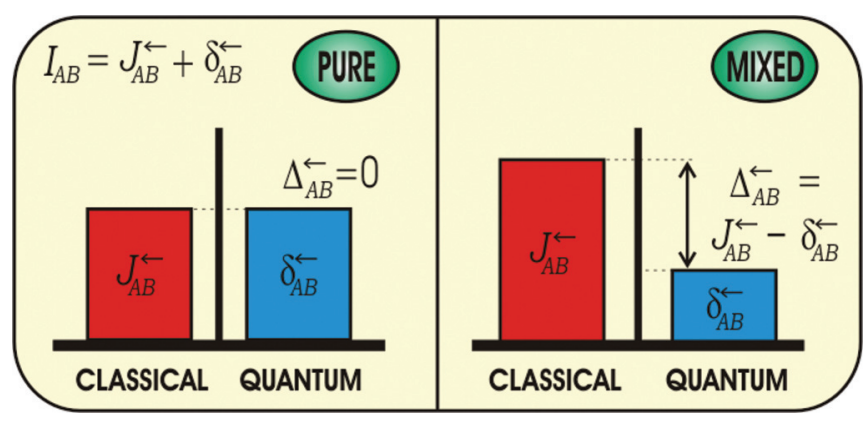

FIG. 1. (Color online) An illustrative scheme of the discrepancy for pure and mixed states. Here, for the mixed state, we just considered in the illustration $J_{A B}^{\leftarrow}>\delta_{A B}^{\overleftarrow{ }}$, but the opposite is also possible. 
more work from correlated systems, in comparison to their classical counterparts, since they have access to nonlocal observables. The difference between their efficiencies is given by the quantum discord which, thus, is always positive. The discrepancy compares this gain with the efficiency of classical demons. If the gain given by the use of global operations does not exceed the work extracted by the local ones, the discrepancy is positive, and it is negative otherwise. In that case, the EOF is monogamous as we will see below. So, in this sense, the discrepancy can be seen as a gain of information when global operations are employed in contrast to local ones. Indeed, by writing

$$
\begin{aligned}
\Delta_{\overparen{A B}}^{\overleftarrow{ }} & =-I_{A B}+2 J_{\overparen{A B}}^{\leftarrow}=-I_{A B}+2 S_{A}-2 S_{q}(A \mid B) \\
& =I_{A B}-2\left[S_{q}(A \mid B)-S(A \mid B)\right],
\end{aligned}
$$

where $S(A \mid B)$ is the conditional entropy and $S_{q}(A \mid B)=$ $\min _{\left\{\Pi_{k}\right\}} \sum_{k} p_{k} S_{A \mid k}$, clarifies the meaning of this interpretation.

We can yet compute the discrepancy $\Delta_{A B}$ as a function of the EOF and the mutual information. Adding and subtracting $I_{A B}$ on the right side of Eq. (2), it is straightforward to show that

$$
\Delta_{\overparen{A B}}^{\leftarrow}=I_{A B}-2 \delta_{A B}^{\leftarrow}
$$

Since $\delta_{A B}$ is related to the EOF of the pair $A C, E_{A C}$ through the relation $[8,19] \delta_{A B}^{\leftarrow}=E_{A C}+S_{A \mid C}$, and the fact that $S(A \mid B)=-S(A \mid C)$ for pure states, we obtain

$$
\Delta_{A B}^{\leftarrow}=I_{A C}-2 E_{A C}
$$

This equation shows that the discrepancy between the pair $A B$ is positive if $I_{A C} / 2>E_{A C}$ and relates the balance between the classical and the quantum correlations to the balance of the entanglement and the mutual information. In fact $I_{A C} / 2$ is a lower bound to the entanglement of purification [20] and so, whenever the EOF is smaller than that, the information gain on using global operations does not exceed the information acquired with local operations only. Finally, the discrepancy also follows a conservation relation: Observing the discrepancy as a function of the EOF and the mutual information, and noting that these quantities are symmetric, i.e., $E_{A C}=E_{C A}$ and $I_{A C}=I_{C A}$, we obtain

$$
\Delta_{\overparen{A B}}^{\leftarrow}=\Delta_{C B}^{\leftarrow}
$$

This equality means that in a pure tripartite joint system the discrepancy of the correlations established with a particular system is constant under a permutation of the complementary subsystems, or that the gain of information on using global operations over local operations is constant under permutation of the complementary subsystems. This fundamental aspect is general, valid for any tripartite pure state, and rules how the classical and quantum correlations are distributed among the parties.

Given the definitions above we now discuss the condition for a monogamous distribution of EOF through the system. We begin by using the conservation law for distribution of the EOF and QD [8]

$$
E_{A B}+E_{A C}=\delta_{A B}^{\overleftarrow{A}}+\delta_{A C}^{\overleftarrow{A}}
$$

$E_{i j}$ being the EOF between subsystems $i$ and $j$. Equation (8) can be rewritten in a different form by considering the EOF between $A$ and the joint system $B C$. Since the tripartite system is pure, we have that $E_{A(B C)}=S_{A}$, the von Neumann entropy of the subsystem $A$. Thus, we can write the relation for distribution of the EOF as

$$
E_{A B}+E_{A C}+\tau_{A}=E_{A(B C)},
$$

where the EOF tangle $\tau_{A}$ is identified as

$$
\tau_{A} \equiv S_{A}-\delta_{\overparen{A B}}^{\leftarrow}-\delta_{A C}^{\leftarrow}
$$

Although the above equation already gives the required tangle of the EOF, it does not elucidate the operational meaning of $\tau_{A}$, which is apparent when we relate it to the classical correlation. Replacing Eq. (2) in Eq. (10), we find that $\tau_{A}=$ $J_{A B}^{\leftarrow}+J_{A C}^{\leftarrow}-S_{A}$, which combined with Eq. (10) results in

$$
\tau_{A}=\frac{1}{2}\left[\Delta_{A B}^{\overleftarrow{A}}+\Delta_{\overparen{A C}}^{\overleftarrow{ }}\right]
$$

This equation shows that the EOF tangle is the average of the correlation discrepancy, thereby giving the imbalance between the classical and quantum correlations. The EOF tangle written in such a form provides the desired operational interpretation in terms of the quantum and classical correlation balance. Moreover, based on this result, it is straightforward to predict when the EOF tangle [Eq. (11)] is negative or not, i.e.,

$$
\tau_{A} \geqslant 0 \quad \text { if and only if } \quad J_{A B}^{\overleftarrow{A}}+J_{A C}^{\overleftarrow{A}} \geqslant \delta_{A B}^{\overleftarrow{A}}+\delta_{\overparen{A C}}^{\leftarrow}
$$

So monogamy of the EOF is equivalent to the inequality $J_{\overparen{A B}}+J_{\overparen{A C}} \geqslant \delta_{\overparen{A B}}+\delta_{\overparen{A C}}$. Explicitly, Eq. (12) shows that whenever the minimized quantum conditional entropies $S_{q}(A \mid i)=\min _{\left\{\Pi_{k}\right\}} \sum_{k} p_{k} S\left(\rho_{A \mid k}\right), \quad \rho_{A \mid k}=$ $\operatorname{Tr}_{i}\left(\Pi_{k}^{i} \rho_{A i} \Pi_{k}^{i}\right) / \operatorname{Tr}_{A i}\left(\Pi_{k}^{i} \rho_{A i} \Pi_{k}^{i}\right), i=B, C$, follow the inequality $S_{A} \geqslant S_{q}(A \mid B)+S_{q}(A \mid C)$, the EOF is monogamous. Note that each of the quantum conditional entropies is upper bounded by $S_{A}$ and so the above is a more restrictive bound. Thus, whenever

$$
S_{A}<S_{q}(A \mid B)+S_{q}(A \mid C) \leqslant 2 S_{A},
$$

the EOF is not a monogamous measure and $\tau_{A}$ is negative, meaning that the use of global operations gives an information gain which is larger than the information acquired with local operations only.

\section{PERMUTATION-INVARIANT TANGLE AND APPLICATIONS}

One can note that the EOF tangle is not invariant under permutations of the subsystems, once $\tau_{A} \neq \tau_{B} \neq \tau_{C}$ in general, for $\tau_{B}=\left(\Delta_{B A}^{\leftarrow}+\Delta_{B C}^{\leftarrow}\right) / 2$, and $\tau_{C}=\left(\Delta_{C B}^{\leftarrow}+\Delta_{C A}^{\leftarrow}\right) / 2$, in contrast to the concurrence tangle for pure states [1]. The reason for that is simple, since the EOF tangle is not a measure of a genuine tripartite entanglement, being a measure of the gain on using global operations over local ones instead. The different $\tau_{A}, \tau_{B}$, and $\tau_{C}$ are related to different instances where the local operations are made. To define an invariant quantity and an essential measure of the global gain in the triple, we use the sum of the distinct EOF tangles. To calculate the sum of the EOF tangles, we use the notation in terms of the flow of quantum correlation [11], defined as

$$
\begin{aligned}
& \mathcal{L}_{\circlearrowright} \equiv \delta_{B A}^{\overleftarrow{ }}+\delta_{\overparen{C B}}^{\overleftarrow{ }}+\delta_{\overparen{A C}}^{\leftarrow}, \\
& \mathcal{L}_{\circlearrowleft} \equiv \delta_{\overparen{C A}}^{\overleftarrow{ }}+\delta_{B C}^{\overleftarrow{B C}}+\delta_{\overparen{A B}}^{\overleftarrow{ }},
\end{aligned}
$$


and the flow of classical correlation

$$
\begin{aligned}
& \mathcal{J}_{\circlearrowright} \equiv J_{B A}^{\leftarrow}+J_{C B}^{\leftarrow}+J_{A C}^{\leftarrow}, \\
& \mathcal{J}_{\circlearrowleft} \equiv J_{C A}^{\leftarrow}+J_{B C}^{\leftarrow}+J_{A B}^{\leftarrow}
\end{aligned}
$$

In Eqs. (14) and (16) the correlations between pairs are computed sequentially as $A \rightarrow B \rightarrow C$, i.e., for the tripartite system $A B C$, we start by doing measurements on $A$ to infer the correlations (quantum and classical) between $A$ and $B$, then we measure $B$ to infer the correlations between $B$ and $C$, and finally we measure $C$ to infer the correlations between $C$ and $A$, representing a clockwise, $\mathcal{L}_{\circlearrowright}$ and $\mathcal{J}_{\circlearrowright}$, flow of information. Conversely, the computation of the correlations in Eqs. (15) and (17) for the sequence $C \rightarrow B \rightarrow A$ represents a counterclockwise flow. We now recall a result given in our previous work [11]. For a general tripartite pure state, $\mathcal{L}_{\circlearrowright}=\mathcal{L}_{\circlearrowleft}$, and thus through Eq. (1) together with the fact that the mutual information is symmetric, $I_{i j}=I_{j i}$, it is straightforward to prove that $\mathcal{J}_{\circlearrowright}=\mathcal{J}_{\circlearrowleft}$. So we can write the sum of the EOF tangles as

$$
\tau_{A B C} \equiv \tau_{A}+\tau_{B}+\tau_{C}=\mathcal{J}_{\circlearrowright}-\mathcal{L}_{\circlearrowright}=\Delta_{\circlearrowright},
$$

where $\Delta_{\circlearrowright} \equiv \Delta_{B A} \leftarrow \Delta_{C B}+\Delta_{A C}$. This equation proves that the flow of the locally accessible information (classical correlation) minus the flow of the inaccessible information (quantum discord), or the clockwise flow of discrepancy, is equal to the sum of the EOF tangles. Indeed $\tau_{A B C}$ is positive whenever the information gain through the usage of global operations is not larger than the information acquired with local operations on every party. It is important to remark that this feature is general and valid for arbitrary tripartite pure states.

\section{TANGLE FOR A $2 \times 2 \times N$ QUANTUM SYSTEM AND ENTANGLEMENT SUDDEN DEATH}

We now exemplify the computation of the EOF tangle for a tripartite pure state with dimensions $2 \times 2 \times N$. This system is particularly interesting since it allows us to characterize how a two-qubit system becomes entangled with an environment. We can rewrite Eq. (18) as

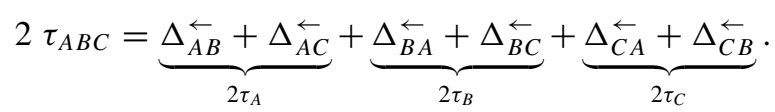

The main obstacle to calculating $\tau_{A B C}$ is to determine the four elements $\Delta_{A C}, \Delta_{B C}^{\leftarrow}, \Delta_{C A}$, and $\Delta_{C B}^{\leftarrow}$, since the elements $\Delta_{A B}^{\leftarrow}$ and $\Delta_{B A}^{\leftarrow}$ can be computed straightforwardly. On the other hand, by examining Eq. (7), we notice that $\Delta_{A C}^{\leftarrow}=\Delta_{B C}^{\leftarrow}$ and both elements can be evaluated through the EOF and the mutual information of the pair $A B$, once $\Delta_{A C}=\Delta_{B C}^{\overleftarrow{ }}=$ $I_{A B}-2 E_{A B}$. Similarly, $\Delta_{C A}^{\leftarrow}=\Delta_{B A}^{\leftarrow}$, while $\Delta_{C B}^{\leftarrow}=\Delta_{A B}^{\overleftarrow{B}}$, and so the resulting EOF tangle is given by $\tau_{A B C}=\Delta_{A B}^{\leftarrow}+\Delta_{B A}^{\leftarrow}+$ $\Delta \overleftarrow{A C}$

To exemplify, we examine how two qubits evolve when coupled to two independent environments at zero temperature, acting as amplitude-damping channels. The effective system in question has minimum dimensions of $2 \times 2 \times 4$ and, as shown above, $\tau_{A B C}$ can be trivially calculated. The model
Hamiltonian can be written as

$$
H=\omega_{0}^{(i)} \sigma_{+}^{(i)} \sigma_{-}^{(i)}+\sum_{k} \omega_{k}^{(i)} a_{k}^{(i) \dagger} a_{k}^{(i)}+\left(\sigma_{+}^{(i)} B^{(i)}+\sigma_{-}^{(i)} B^{(i)^{\dagger}}\right)
$$

where $B^{(i)}=\sum_{k} g_{k}^{(i)} a_{k}^{(i)}$ with $g_{k}^{(i)}$ being the coupling constant, $\omega_{0}^{(i)}$ is the transition frequency of the $i$ th qubit, and $\sigma_{ \pm}^{(i)}$ are the $i$ th qubit's raising and lowering operators. The index $k$ labels the reservoir field modes with frequencies $\omega_{k}^{(i)}$, and $a_{k}^{(i)_{\dagger}}\left(a_{k}^{(i)}\right)$ is the usual creation (annihilation) operator. We suppose that the environments are represented by a bath of harmonic oscillators, and the spectral density is of the form $j(\omega)=\gamma_{0} \lambda^{2} / 2 \pi\left[\left(\omega_{0}-\omega\right)^{2}+\lambda^{2}\right]$, where $\lambda$ is connected to the reservoir correlation time and $\gamma_{0}$ is related to the time scale over which the state of the system changes.

Entanglement sudden death (ESD) is a characteristic phenomenon occurring in multipartite open quantum systems: Depending on the initial conditions and the interaction between the system and its environment, an initial amount of entanglement can vanish at a finite time. It occurs whenever each system partition is subjected to local channels, and whether the dynamics is Markovian or non-Markovian. Here, we treat non-Markovian dynamics only and, in this situation, depending on the system initial conditions, two distinct types of ESD can be observed: (i) during the dynamics, entanglement vanishes during a finite time interval; and (ii) the entanglement vanishes just at specific discrete times. The first case will be called here genuine ESD since even for a Markovian approach, the ESD phenomenon is yet present. In a different way, in case (ii) the ESD is not present for a Markovian approach.

In Fig. 2(a) we consider an initial Bell-like state for the two qubits $A$ and $B:|\psi\rangle=a|00\rangle+\sqrt{1-a^{2}}|11\rangle$, with $a=1 / \sqrt{3}$, which is a typical situation where the entanglement between $A$ and $B$ (dashed line in green) suddenly disappears [21]. On the other hand, in Fig. 2(b) we take $a=\sqrt{2 / 3}$ where $E_{A B}$ is null only at some discrete times. As mentioned above, this last situation would not show the ESD phenomenon
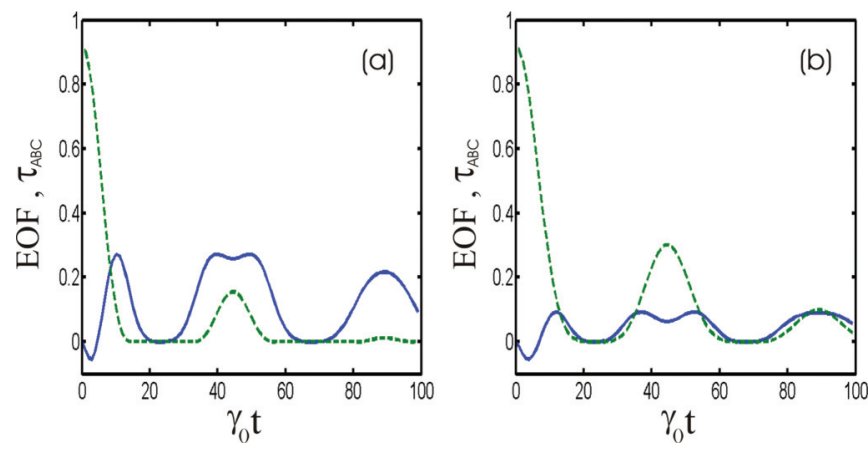

FIG. 2. (Color online) The EOF dynamics between the qubits $A$ and $B$ is represented by the dashed line (green) and the $\tau_{A B C}$ dynamics is represented by the solid line (blue). The parameter adopted for the initial state in (a) is $a=1 / \sqrt{3}$, while in (b) we use $a=\sqrt{2 / 3}$. We suppose a strong coupling in both cases with $\lambda=0.01 \gamma_{0}$. When the bipartite entanglement $E_{A B}$ tends to vanish, $\tau_{A B C}$ tends to a local positive maximum, indicating a monogamic redistribution of entanglement at those points. This is nonetheless not generally monogamous since there are intervals of time (at the beginning of the dynamical evolution) where $\tau_{A B C}$ is negative. 
were we considering a Markovian dynamics. We see in both Figs. 2(a) and 2(b) that the tangle $\tau_{A B C}$ (solid line in blue) quickly becomes positive, meaning that the environment $C$ induces the global state to converge in one state that satisfies the monogamous relation. This characteristic occurs because the QD tends to decay while the classical correlation tends to be stable, at least after the transition where the classical correlation becomes greater than the quantum one [22]. More importantly, we might establish a connection between the ESD phenomenon and the balance of classical and quantum correlations. To understand this characteristic, we must reconsider Eq. (9). When the entanglement between $A B$ vanishes, the entanglement between the qubit $A$ and the environment $\left(E_{A C}\right)$ must be equal to $E_{A(B C)}-\tau_{A}$ (here the subsystem $C$ represent the environment). Moreover, when $a=\sqrt{2 / 3}$, Fig. 2(b) shows that $E_{A B}=0$ just when $E_{A C}=E_{A(B C)}$ since the system $A C$ becomes pure and thus $\tau_{A}$ must vanish. In this case, the entanglement suddenly disappears when the discrepancy in the global system (qubits plus environment) is zero, or when the information gain in using global operations is equal to the information acquired by using local operations in $B$ and $C$ only.

\section{MIXED TRIPARTITE STATES AND SQUASHED ENTANGLEMENT}

To finalize we sketch an extension for a tripartite mixed state. The EOF tangle can be positive or negative corresponding respectively to the GHZ and $W$ entanglements [13]. In a mixed state, the ensemble may have elements of both families and our aim, in defining a tripartite measure, must be minimizing these two components over all the ensembles. For this purpose, we define two quantities,

$$
\begin{gathered}
\tau_{\mathrm{GHZ}}^{(A)}(|\psi\rangle)=\max \left\{0, \tau_{A}\right\}, \\
\tau_{W}^{(A)}(|\psi\rangle)=\max \left\{0,-\tau_{A}\right\},
\end{gathered}
$$

for any pure tripartite state $|\psi\rangle$. In this way, these measures account for the amount of GHZ and $W$ entanglement in $|\psi\rangle$, Also, they are mutually exclusive in the sense that, if one is larger than zero, the other one is null. Therefore, for mixed states, we can define $\tau_{A}$ as

$$
\tau_{A}(\rho)=\min _{\mathcal{E}}\left\{\sum_{i} p_{i}\left[\tau_{\mathrm{GHZ}}^{(A)}\left(\left|\phi_{i}\right\rangle\right)+\tau_{W}^{(A)}\left(\left|\phi_{i}\right\rangle\right)\right]\right\}
$$

where the minimization goes over all ensembles $\mathcal{E}$ such that $\rho=\sum_{i} p_{i}\left|\phi_{i}\right\rangle\left\langle\phi_{i}\right|$. Obviously, we can also define the corresponding quantities $\tau_{B}$ and $\tau_{C}$ similarly with respect to the subsystems $B$ and $C$.

Now we discuss an issue related to mixed states with more general relevance for entanglement theory. It is known that under some special conditions the EOF is equivalent to the squashed entanglement (SE) [15], a monogamous entanglement measure [19], but it is not clear that all the monogamous distribution of EOF is related to the SE as well. To investigate this relation we note that the SE is defined as

$$
E_{s q}\left(\rho_{A B}\right)=\frac{1}{2} \inf _{\varrho_{A B C} \in K} S(A: B \mid C),
$$

where $K$ is the set of all density matrices $\rho_{A B C}: \rho_{A B}=$ $\operatorname{Tr}_{C} \rho_{A B C}$, and $S(A: B \mid C)$ is the conditional mutual information for the extended system $\rho_{A B C}$. It is known that the SE is upper bounded by the EOF, $E_{\mathrm{sq}}\left(\rho_{A B}\right) \leqslant E_{A B}$. By Eq. (9) for a pure $A B C$ system if EOF is monogamous,

$$
E_{A B}+E_{A C}=E_{A B}+\delta_{A B}^{\leftarrow}+S(A \mid B) \leqslant S_{A},
$$

and since $S_{A}=S(A: B)+S(A \mid B)$, we have

$$
E_{A B}+\delta_{A B}^{\leftarrow} \leqslant S(A: B)
$$

for $\rho_{A B C}$ pure, when $S(A: B \mid C)=S(A: B)$. In fact for arbitrary mixed states $S(A: B \mid C)$ can be larger or smaller than $S(A: B)$, depending on the kind of correlations allowed for the state. So it turns out that for arbitrarily mixed $\rho_{A B C}$, there are instances for which $S(A: B \mid C) \geqslant S(A: B)$ that certainly $S(A: B \mid C) \geqslant E_{A B}+\delta_{A B}$. There are also other instances, for which $S(A: B \mid C) \leqslant S(A: B)$, where either $S(A: B \mid C) \geqslant$ $E_{A B}+\delta_{A B}^{\leftarrow}$ or $S(A: B \mid C) \leqslant E_{A B}+\delta_{A B}^{\leftarrow}$, such that the EOF can be larger or smaller than $\mathrm{QD}$, in distinct instances. So when the EOF is monogamous (and consequently the QD) there are instances, for arbitrarily mixed states $\rho_{A B C}$, where

$$
E_{s q}\left(\rho_{A B}\right) \leqslant \frac{1}{2}\left(E_{A B}+\delta_{\overparen{A B}}^{\leftarrow}\right)
$$

and other distinct instances where

$$
E_{s q}\left(\rho_{A B}\right) \geqslant \frac{1}{2}\left(E_{A B}+\delta_{\overparen{A B}}^{\leftarrow}\right)
$$

where we profited from the fact that the minimization over $C$ does not affect the right-hand side of the inequalities. To simplify the discussion we can check what occurs to states saturating both (22) and (23). There the SE is always related not only to the EOF but to the QD of the pair $A B$ as well. Interestingly when the reduced state $\rho_{A B}$ is pure we recover the well-known results that the $\mathrm{SE}$ is equal to the EOF. But that is also true when there is full permutation invariance on $\rho_{A B C}, \delta_{A B}^{\leftarrow}=E_{A B}$. What is known is that when the set of states that minimize SE is constrained by states of the form $\rho_{A B C}=\sum_{k}\left|\psi_{A B}^{k}\right\rangle\left\langle\psi_{A B}^{k}|\otimes| k\right\rangle\langle k|$, it corresponds to the ensemble minimization employed for the derivation of the EOF, and so the SE is equal to the EOF. We see that this set corresponds to the states saturating both (22) and (23) only when there is total permutation invariance of $A B C$.

As a complement, when the EOF is not monogamous, $E_{A B}+\delta_{A B}^{\leftarrow}+S(A \mid B)>S_{A}$, and we always get that for arbitrarily mixed states $\rho_{A B C}$

$$
E_{s q}\left(\rho_{A B}\right)<\frac{1}{2}\left(E_{A B}+\delta_{\overparen{A B}}^{\leftarrow}\right)
$$

never attaining equality.

\section{DISCUSSION}

To conclude we have discussed an important and simple question: What is the amount of entanglement between $A$ and $B C$ that cannot be accounted for by the entanglement of $A$ with $B$ and $C$ separately? When the entanglement is measured by concurrence, $\mathrm{CKW}$ showed that this amount is always positive. On the other hand the EOF can assume either positive or negative values, depending on the gain that global operations give over local operations. The reason for the negative signal of the EOF tangle, rather than being a failure to take into account a "residual entanglement," is clarified in terms of 
the discrepancy between classical and quantum correlations. We have demonstrated the necessary conditions for this EOF tangle to be positive by considering an arbitrary pure tripartite system. Furthermore, the discrepancy exhibits a conservative relation because one particular subsystem shares always the same amount of discrepancy with other complementary subsystems. This description allows the study of the EOF tangle for a three-qubit system in a very simple manner. Moreover, we described a simple numerical procedure to calculate the EOF tangle for an arbitrary tripartite system with dimensions $2 \times 2 \times N$, which enables the possibility of studying the EOF tangle between two qubits and an environment with $N$ degrees of freedom. In this sense, by examining two qubits coupled to independent amplitude-damping channels, we show that the ESD phenomenon is connected to the discrepancy of the global system. We believe that this relation may allow a deep understanding of the distribution of entanglement and correlation in multipartite systems. In that respect it would certainly be interesting to check the instances indicating polygamy of entanglement [23] in terms of entanglement of assistance [24] in view of the present approach.

\section{ACKNOWLEDGMENTS}

This work is supported by FAPESP and CNPq through the National Institute for Science and Technology of Quantum Information (INCT-IQ). M.C.O. also acknowledges support by iCORE.
[1] V. Coffman, J. Kundu, and W. K. Wootters, Phys. Rev. A 61, $052306(2000)$

[2] T. J. Osborne and F. Verstraete, Phys. Rev. Lett. 96, 220503 (2006).

[3] T. R. de Oliveira, Phys. Rev. A 80, 022331 (2009).

[4] M. F. Cornelio and M. C. de Oliveira, Phys. Rev. A 81, 032332 (2010).

[5] J. S. Kim and B. C. Sanders, J. Phys. A 43, 445305 (2010).

[6] C. H. Bennett, D. P. DiVincenzo, J. A. Smolin and W. K. Wootters, Phys. Rev. A 54, 3824 (1996); M. F. Cornelio, M. C. de Oliveira, and F. F. Fanchini, Phys. Rev. Lett. 107, 020502 (2011).

[7] Here an "extended" state is to be considered as a system given by the original system $A B$ plus the "extension" $C$, so that $\rho_{A B}=$ $\operatorname{Tr}_{C} \rho_{A B C}$.

[8] F. F. Fanchini, M. F. Cornelio, M. C. de Oliveira, and A. O. Caldeira, Phys. Rev. A 84, 012313 (2011).

[9] L. Henderson and V. Vedral, J. Phys. A 34, 6899 (2001).

[10] V. Vedral, Phys. Rev. Lett. 90, 050401 (2003); B. Groisman, S. Popescu, and A. Winter, Phys. Rev. A 72, 032317 (2005); M. Horodecki, P. Horodecki, R. Horodecki, J. Oppenheim, A. Sen, U. Sen, and B. Synak-Radtke, ibid. 71, 062307 (2005).

[11] F. F. Fanchini, L. K. Castelano, M. F. Cornelio, and M. C. de Oliveira, New J. Phys. 14, 013027 (2012).

[12] G. L. Giorgi, Phys. Rev. A 84, 054301 (2011).

[13] R. Prabhu, A.K. Pati, A. SenDe, and U. Sen, Phys. Rev. A 85, 040102(R) (2012).
[14] J. Maziero, L. C. Celeri, R. M. Serra, and V. Vedral, Phys. Rev. A 80, 044102 (2009).

[15] M. Christandl and A. Winter, J. Math. Phys. 45, 829 (2004).

[16] H. Ollivier and W. H. Zurek, Phys. Rev. Lett. 88, 017901 (2001).

[17] W. H. Zurek, Phys. Rev. A 67, 012320 (2003).

[18] J. C. Maxwell, Theory of Heat, 4th ed. (Longmans, Green, London, 1985), pp. 328-329.

[19] M. Koashi and A. Winter, Phys. Rev. A 69, 022309 (2004).

[20] B. M. Terhal, M. Horodecki, D. W. Leung, and D. P. DiVincenzo, J. Math. Phys. 43, 4286 (2002).

[21] K. Zyczkowski, P. Horodecki, M. Horodecki, and R. Horodecki, Phys. Rev. A 65, 012101 (2001); L. Diósi, in Irreversible Quantum Dynamics, Lecture Notes in Physics Vol. 622, edited by F. Benatti and R. Floreanini (Springer, Berlin, 2003), p. 157; P. J. Dodd and J. J. Halliwell, Phys. Rev. A 69, 052105 (2004); T. Yu and J. H. Eberly, Phys. Rev. Lett. 93, 140404 (2004); M. P. Almeida, F. de Melo, M. Hor-Meyll, A. Salles, S. P. Walborn, P. H. Souto Ribeiro, and L. Davidovich, Science 316, 579 (2007).

[22] M. F. Cornelio, O. J. Farias, F. F. Fanchini, I. Frerot, G. H. Aguilar, M. O. Hor-Meyll, M. C. de Oliveira, S. P. Walborn, A. O. Caldeira, and P. H. Ribeiro, Phys. Rev. Lett. 109, 190402 (2012).

[23] F. Buscemi, G. Gour, and J. S. Kim, Phys. Rev. A 80, 012324 (2009).

[24] G. Gour, D. A. Meyer, and B. C. Sanders, Phys. Rev. A 72, 042329 (2005) 